A. KuROSCH

1. Theory of groups (in Russian), Moscow, 1944.

Marston Morse and G. A. Hedlund

1. Symbolic dynamics, Amer. J. Math. vol. 60 (1938) p. 815.

G. T. WHYBURN

1. Analytic topology, Amer. Math. Soc. Colloquium Publications, vol. 28, New York, 1942.

The University of Puerto Rico and

THE UNIVERSITY OF VIRGINIA

\title{
A ZERO-DIMENSIONAL TOPOLOGICAL GROUP WITH A ONE-DIMENSIONAL FACTOR GROUP
}

\section{SAMUEL KAPLAN}

As can be easily shown, if a locally compact topological group is zero-dimensional, all of its factor groups are zero-dimensional. In this note we give an example of a non locally compact zero-dimensional group with a factor group which is topologically isomorphic to the real numbers, hence one-dimensional. ${ }^{1}$

1. Preliminaries. Let $\{\lambda\}$ be a set of indices of cardinality $c$, and for each $\lambda$, let $R_{\lambda}$ be a topological isomorph of the additive group of rational numbers. We form the weak product $R$ of the $R_{\lambda}$ : an element $r$ of $R$ is a collection $r=\left\{r_{\lambda}\right\}, r_{\lambda} \in R_{\lambda}$, such that for only a finite number of the $\lambda^{\prime}$ s is $r_{\lambda} \neq 0_{\lambda}$. Under the definitions $r+r^{\prime}=\left\{r_{\lambda}+r_{\lambda}^{\prime}\right\}$, $0=\left\{0_{\lambda}\right\}, R$ forms a group.

Now for each $r \in R$, we define $\|r\|=\sum_{\lambda}\left|r_{\lambda}\right|$. Since all but a finite number of the $r_{\lambda}=0_{\lambda}$, this sum exists. Clearly $\left\|r+r^{\prime}\right\| \leqq\|r\|+\left\|r^{\prime}\right\|$, and $\|-r\|=\|r\|$, hence, as can be easily shown, $\|r\|$ defines a metric in $R$ under the definition: the distance from $r$ to $r^{\prime}$ is $\left\|r-r^{\prime}\right\|$.

Lemma 1. Let $\left\{d_{\lambda}\right\}$ be a set of positive real numbers bounded away from zero, that is, there exists $d>0$ such thal $d_{\lambda} \geqq d$ for all $\lambda$. Then

$$
U=\left\{r\left|\sum_{\lambda}\right| \frac{r_{\lambda}}{d_{\lambda}} \mid<1\right\}
$$

Received by the editors November $10,1947$.

${ }^{1}$ Cf. Bourbaki, Topologie generale, chap. III, p. 21, exercise 12, for an example of a totally disconnected group with a factor group topologically isomorphic to the reals. This example was pointed out to me by I. Kaplansky. 
is an open set containing the origin, and

$$
\bar{U} \subset\left\{r\left|\sum_{\lambda}\right| \frac{r_{\lambda}}{d_{\lambda}} \mid \leqq 1\right\} .
$$

PROOF. We need only prove that the real valued function $f(r)$ $=\sum_{\lambda}\left|r_{\lambda} / d_{\lambda}\right|$ is continuous. Given $r=\left\{r_{\lambda}\right\}$ and $\epsilon>0$, choose $\delta<d \epsilon$. Now, for any $r^{\prime}=\left\{r_{\lambda}^{\prime}\right\}$,

$$
\begin{aligned}
\left|\sum_{\lambda}\right| \frac{r_{\lambda}^{\prime}}{d_{\lambda}}\left|-\sum_{\lambda}\right| \frac{r_{\lambda}}{d_{\lambda}}|| & =\left|\sum_{\lambda} \frac{\left|r_{\lambda}^{\prime}\right|-\left|r_{\lambda}\right|}{d_{\lambda}}\right| \leqq \sum_{\lambda}\left|\frac{\left|r_{\lambda}^{\prime}\right|-\left|r_{\lambda}\right|}{d_{\lambda}}\right| \\
& \leqq \frac{1}{d} \sum_{\lambda}|| r_{\lambda}^{\prime}|-| r_{\lambda}|| \\
& \leqq \frac{1}{d} \sum_{\lambda}\left|r_{\lambda}^{\prime}-r_{\lambda}\right| \\
& =\frac{1}{d}\left\|r^{\prime}-r\right\| .
\end{aligned}
$$

Thus, if $\left\|r^{\prime}-r\right\|<\delta$, this last expression is less than $\epsilon$, which proves the continuity and hence the lemma.

LEMMA 2. Let $\left\{\alpha_{\lambda}\right\}$ be a bounded set of positive irrational numbers linearly independent with respect to the rationals, that is:

$$
a_{1} \alpha_{\lambda(1)}+\cdots+a_{h} \alpha_{\lambda(h)}=a \quad\left(a_{1}, \cdots, a_{h}, a \text { rational }\right)
$$

implies

$$
a_{1}=\cdots=a_{h}=a=0 .
$$

Then if, in Lemma 1 , we take $d_{\lambda}=1 / \alpha_{\lambda}$, the resulting $U$ has a vacuous boundary.

Proof. From the second part of Lemma 1, we need only prove that there is no $r$ such that $\sum_{\lambda}\left|r_{\lambda} / d_{\lambda}\right|=1$. Assume there is. Then, since replacing an $r_{\lambda}$ by its negative does not change absolute values, we can assume all the $r_{\lambda}$ are non-negative. Then we have $\sum_{\lambda} r_{\lambda} / d_{\lambda}=1$. But each $d_{\lambda}=1 / \alpha_{\lambda}$, hence $\sum_{\lambda} r_{\lambda} \alpha_{\lambda}=1$, which contradicts the hypothesis on the $\alpha_{\lambda}$ 's.

Using Lemma 2, we now define a special sequence of neighborhoods $\left\{U_{n}\right\}(n=0,1, \cdots)$ which form a basis around the origin. We first take the set of real numbers $1 / 2 \leqq c<1$ and set them in one-one correspondence with the $\lambda$ 's: $\left\{c_{\lambda}\right\}$. (Our purpose in bringing these in will 
become clear in \$2.) We then choose a set of irrational numbers $\left\{\alpha_{\lambda}\right\}$ with the property (1) (the existence of such a set follows from the existence of a Hamel basis for the reals), and such that for each $\lambda, c_{\lambda}<\alpha_{\lambda}<1$. This last can always be accomplished by multiplying $\alpha_{\lambda}$ by a suitable rational. We then have

$$
1 / 2 \leqq c_{\lambda}<\alpha_{\lambda}<1
$$

for all $\lambda$.

Now for each $n$, we define $U_{n}$ by taking

$$
d_{\lambda}^{(n)}=\frac{1}{2^{n}} \cdot \frac{1}{\alpha_{\lambda}},
$$

and letting

$$
U_{n}=\left\{r\left|\sum_{\lambda}\right| \frac{r_{\lambda}}{d_{\lambda}{ }^{\prime}} \mid<1\right\} .
$$

Since, for $r \in U_{n}$,

$$
\|r\|=\sum_{\lambda}\left|r_{\lambda}\right|=\sum_{\lambda} d_{\lambda}^{(n)}\left|\frac{r_{\lambda}}{d_{\lambda}^{(n)}}\right| \leqq \frac{2}{2^{n}} \sum_{\lambda}\left|\frac{r_{\lambda}}{d_{\lambda}^{(n)}}\right|<\frac{1}{2^{n-1}},
$$

the diameter of $U_{n}$ is less than $1 / 2^{n-2}$, hence approaches zero as $n$ goes to infinity. Thus $\left\{U_{n}\right\}$ constitutes a basis around the origin. Since, from Lemma 2, the boundary of each $U_{n}$ is vacuous, it follows that $R$ is zero-dimensional.

2. The example. Let $R_{*}$ be the additive group of real numbers $\left\{r_{*}\right\}$ with distance defined by $\left\|r_{*}\right\|=1$ for all $r_{*}$ different from zero. This makes it discrete. Let $G=R_{*} \times R$ with distance defined as follows: If $g=\left(r_{*}, r\right)$ then $\|g\|=\left\|r_{*}\right\|+\|r\|$. Since $R_{*}$ is discrete, the $U_{n}$ 's, now considered as subsets of $G$, form a basis around the origin of $G$, hence $G$ is zero-dimensional.

We define the subgroup $H$ of $G$ as the set of all $g=\left(r_{*}, r\right)$ such that $r_{*}+\sum_{\lambda} c_{\lambda} r_{\lambda}=0$ (cf. (2)).

\section{LEMMA 3. $H$ is a closed subgroup of $G$.}

PRoof. $H$ is a subgroup, for if $g, g^{\prime} \in H$ then $r_{*}+\sum_{\lambda} c_{\lambda} r_{\lambda}=0$ and $r_{*}^{\prime}+\sum_{\lambda} c_{\lambda} r_{\lambda}^{\prime}=0$, hence $\left(r_{*}-r_{*}^{\prime}\right)+\sum_{\lambda} c_{\lambda}\left(r_{\lambda}-r_{\lambda}^{\prime}\right)=0$. To prove $H$ is closed, it is sufficient to show that the real-valued function $f(g)=r_{*}$ $+\sum_{\lambda} c_{\lambda} r_{\lambda}$ is continuous. Given $g=\left(r_{*}, r\right)$ and $\epsilon>0$, choose $\delta<\min (\epsilon, 1)$. Consider any $g^{\prime}=\left(r_{*}^{\prime}, r^{\prime}\right)$ such that $\left\|g^{\prime}-g\right\|<\delta$. We note first that $r_{*}^{\prime}=r_{*}$, for otherwise $\left\|r_{*}^{\prime}-r_{*}\right\|=1$ and hence $\left\|g^{\prime}-g\right\|$ $\geqq 1 \geqq \delta$. Then 


$$
\begin{aligned}
\left|r_{*}^{\prime}+\sum_{\lambda} c_{\lambda} r_{\lambda}^{\prime}-r_{*}-\sum_{\lambda} c_{\lambda} r_{\lambda}\right| & =\left|\sum_{\lambda} c_{\lambda} r_{\lambda}^{\prime}-\sum_{\lambda} c_{\lambda} r_{\lambda}\right| \\
& \leqq \sum_{\lambda} c_{\lambda}\left|r_{\lambda}^{\prime}-r_{\lambda}\right| \\
& \leqq \sum\left|r_{\lambda}^{\prime}-r_{\lambda}\right| \\
& =\left\|g^{\prime}-g\right\|<\delta<\epsilon .
\end{aligned}
$$

This proves the lemma.

LEMMA 4. $G / H$ is algebraically isomorphic to the real numbers.

Proof. Since $R_{*} \times 0 \subset G$ is algebraically isomorphic to the real numbers, it is sufficient to prove that $G / H$ is algebraically isomorphic to this subgroup.

(i) Every coset of $H$ contains an element of $R_{*} \times 0$.

For, let $g=\left(r_{*}, r\right)$ be any element of $G$, where $r=\left\{r_{\lambda}\right\}$. Then if $g^{\prime}=\left(r_{*}+\sum_{\lambda} c_{\lambda} r_{\lambda}, 0\right), g^{\prime}-g=\left(\sum_{\lambda} c_{\lambda} r_{\lambda},-r\right) \in H$. Since $g^{\prime} \in R_{*} \times 0$, this proves (i).

(ii) Different elements of $R_{*} \times 0$ lie in different cosets.

For, let $g=\left(r_{*}, 0\right), g^{\prime}=\left(r_{*}^{\prime}, 0\right)$, with $r_{*} \neq r_{*}^{\prime}$. Then $g^{\prime}-g=\left(r_{*}^{\prime}-r_{*}, 0\right)$, and since $\left(r_{*}^{\prime}-r_{*}\right)+0 \neq 0, g^{\prime}-g \in H$. This proves (ii), and with it, Lemma 4.

We can thus denote each element of $G / H$ by a unique real number. From the proof of (i) above, we see that the real number is given by the mapping

$$
\pi\left(r_{*}, r\right)=r_{*}+\sum_{\lambda} c_{\lambda} r_{\lambda}
$$

LEMma 5. $\pi\left(U_{n}\right)=\left[-1 / 2^{n}<x<1 / 2^{n}\right]$ for all $n=0,1, \cdots$.

PROOF. Since the argument is the same for all $n$, it is sufficient to prove this for $U_{0}$. From (4) and (3),

$$
U_{0}=\left\{g=(0, r)\left|\sum_{\lambda}\right| \alpha_{\lambda} r_{\lambda} \mid<1\right\} \text {. }
$$

(i) $\pi\left(U_{0}\right) \subset[-1<x<1]$.

For, if $g=(0, r) \in U_{0}$, then from (5),

$$
\begin{aligned}
|\pi(g)|=\left|\sum_{\lambda} c_{\lambda} r_{\lambda}\right| & \leqq \sum_{\lambda}\left|c_{\lambda} r_{\lambda}\right| \\
& <\sum_{\lambda}\left|\alpha_{\lambda} r_{\lambda}\right| \\
& <1
\end{aligned}
$$


(ii) $[-1<x<1] \subset \pi\left(U_{0}\right)$.

For consider any real number $x$ such that $-1<x<1$. Since the $c_{\lambda}$ 's run through all the real numbers from $1 / 2$ to 1 , there is a $c_{\lambda^{\prime}}$ such that

$$
x=\epsilon C_{\lambda^{\prime}},
$$

where $\epsilon$ is one of the values $\pm 1, \pm 1 / 2$. Hence, if $g$ is the element of $G$ whose $\lambda^{\prime}$-coordinate is $\epsilon$ and whose remaining coordinates are 0 , we have from (5) that

$$
\pi(g)=c_{\lambda^{\prime} \epsilon}=x .
$$

Thus $x$ has an inverse in $U_{0}$ under $\pi$. Since $x$ was any element of $[-1<x<1]$, (ii) is proved. This establishes the lemma.

Since the set $\left\{U_{n}\right\}$ is a basis around the origin of $G$, the set $\left\{\pi U_{n}\right\}$ is by definition a basis around zero in $G / H$. Hence, from Lemma 5 , $G / H$ has the topology of the real numbers.

Institute for Advanced Study 